\title{
A New Adaptive Algorithm for Digital Predistortion Using LS with Singular Value Decomposition
}

\author{
Qin Zhang \\ Engineering Research Center of Digital Audio \& Video, \\ Ministry of Education \\ Communication University of China \\ Beijing, China \\ zhangqin0425@cuc.edu.cn
}

\author{
Zhibin Zeng \\ Engineering Research Center of Digital Audio \& Video, \\ Ministry of Education \\ Communication University of China \\ Communication University
Beijing, China
zhbzeng@cuc.edu.cn \\ Communication University o
Beijing, China
zhbzeng@cuc.edu.cn
}

\begin{abstract}
Adaptive algorithm plays an important role in digital predistortion for the linearization of power amplifiers. In this paper, a new approach which uses least square (LS) adaptive algorithm with singular value decomposition (SVD) is presented. When calculating inversion matrix of power amplifier model, if combined with singular value decomposition, LS can not only excavate important structural information of the matrix, but also reduce the dimension of the matrix, and therefore, improve the stability and decrease computation complexity.
\end{abstract}

Keywords-power amplifier; digital predistortion; LS; SVD

\section{INTRODUCTION}

Power amplifier (PA) is an indispensable part of transmitting system for wireless communication. Wide-band signals, especially with high peak-to-average power ratio (PAPR), however, will always produce nonlinearity, thus causing spectral broadening as well as in-band distortion when passing through PA, which will degrade adjacent channel power ratio (ACPR) [1] and receiver error rate.

In order to achieve greater efficiency by operating at higher output power while still maintaining spectral compliance, reducing system capital and operational expenditure, there are various methods for linearization of power amplifier [2], such as power back-off, feed-forward method, negative feedback and digital predistortion. Digital predistortion technology is widely used in modern wireless communication by its high precision, strong adaptability and simple implementation. On the other hand, in order to adapt to the nonlinear characteristics of the different power amplifier, adaptive algorithm such as LS and LMS, is indispensable and plays a central role in the digital predistortion system. However, LS algorithm has the disadvantage of random perturbations update weight values. The singular value decomposition is the most reliable decomposition method because of its characteristics of matrix dimension reduction, scale invariance and matrix perturbation insensitivity.

Volterra series is the most frequently used complex series to model power amplifiers. For simplicity, Volterra series is usually deployed in its simplified form, for example, memory polynomial.

\section{ADAPTIVE ALGORITHM}

\section{A. DPD Overview}

The nonlinear characteristics of power amplifier can be represented by their amplitude modulation to amplitude modulation conversion (AM/AM) and amplitude modulation to phase modulation conversion (AM/PM). The essence of digital predistortion [3] is that the amplitude and phase of input signals are preprocessed to compensate the $\mathrm{AM} / \mathrm{AM}$ and $\mathrm{AM} / \mathrm{PM}$ distortion brought by power amplifier. As shown in Figure 1, the predistorter (PD) is added in the front of the power amplifier (PA) of a nonlinear device with extended nonlinear characteristics just opposite to the nonlinear characteristics of power amplifier.
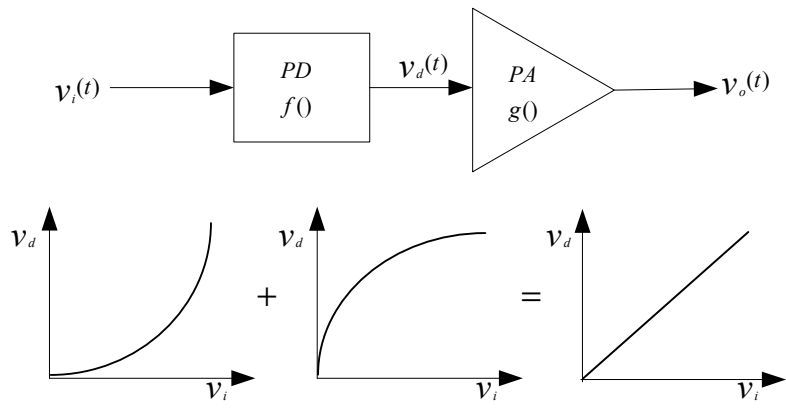

Figure 1. The predistortion basic schematic

\section{B. Predistorter Architecture}

Predistortion system architectures are divided into two types. One is direct learning structure, which must identify the PA nonlinear characteristics and then find the inverse characteristics of PA, which is of high computational complexity. Another type is the indirect learning structure [4], which doesn't need to find the inverse nonlinear characteristics of PA. As a result, the indirect learning structure is more attractive to the adaptive algorithm.

The block diagram of indirect learning is demonstrated in Figure 2. It can be seen that adaptive algorithm updates the predistortion coefficients by comparing the error between the input and output samplings until the error equal or approximate to zero [5]. 


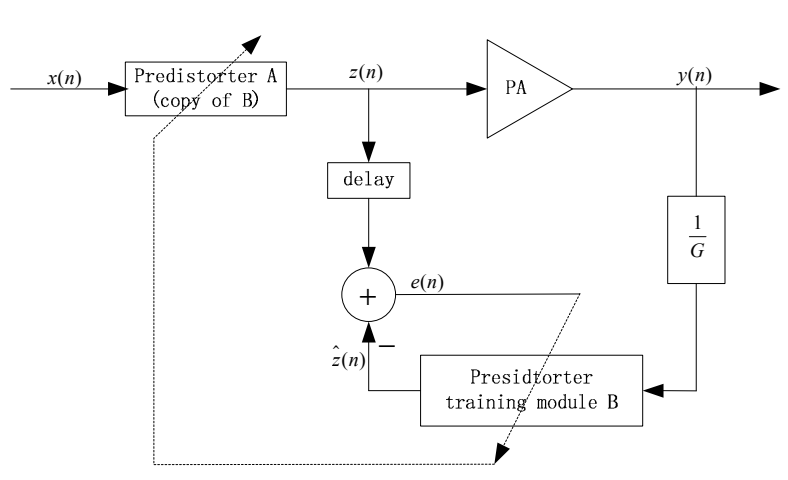

Figure 2. Indirect learning architecture block diagram

\section{Power Amplifier Modelling}

Volterra series is a kind of functional series and can be used to represent memory effects of nonlinear systems with the following expression [6]:

$$
z(t)=\sum_{n=1}^{\infty} \frac{1}{n !} \int_{-\infty}^{\infty} d u_{1} \ldots \int_{-\infty}^{\infty} d u_{n} g_{n}\left(u_{1}, \ldots, u_{n}\right) \prod_{r=1}^{\pi} x\left(t-u_{r}\right)
$$

Where $x(\mathrm{t})$ and $z(\mathrm{t})$ denote the input and output of PA, respectively; $g_{n}\left(u_{1}, \ldots, u_{n}\right)$ is the nth-order Volterra kernel.

But because of its high algorithm complexity, and rising exponentially with the order of polynomial and memory depth, so it is difficult to directly using for high order with memory predistorter for the design and implementation. This article uses the simplified model of the series -- memory polynomial [7] with its expression given by:

$$
z(n)=\sum_{k=1}^{K} \sum_{q=0}^{Q} a_{k q} x(n-q)|x(n-q)|^{k-1}
$$

In this equation, $a_{k, q}$ is the complex coefficients of polynomial term, $K$ the polynomial order, and $Q$ the memory depth.

\section{SVD Profile}

Define $A \in R^{m \times n}$, a non-negative square root of $A^{T} A$ characteristic value are referred as the singular values of A, singular value of the whole referred as $\sigma(A)$. When A is a complex matrix $C^{m \times n}, A^{T} A$ is expressed as $A^{H} A$.

Theorem 1 (Singular Value Decomposition Theorem): If matrix $A \in R^{m \times n}$, there must be two orthogonal matrixes [8]

$$
\begin{aligned}
& U=\left[u_{1}, \cdots, u_{m}\right] \in R^{m \times n} \\
& V=\left[v_{1}, \cdots, v_{n}\right] \in R^{m \times n}
\end{aligned}
$$

And $U$ and $V$ satisfy the following equation:

$$
U^{T} A V=\underset{r}{\left[\begin{array}{cc}
\sum_{r} & 0 \\
0 & 0
\end{array}\right]_{m-r}^{r}}
$$

Where

$\Sigma_{r}=\operatorname{diag}\left(\sigma_{1}, \cdots, \sigma_{r}\right), \sigma_{1} \geq \cdots \geq \sigma_{r}>0[9]$

$\sigma_{i}:$ the singular values of $A$

$u_{i}: i$-th left singular vector

$v_{i}: i$-th right singular vector

As for complex matrix $A \in C^{m \times n}$, just put $U, V$ into unitary matrix, Theorem 1 still holds [8].

For dimension reduction, the singular value decomposition of the matrix $A_{m \times n}$ can be understood as a linear transformation. When n-dimensional space is mapped to r-dimensional space, the singular vectors $u_{i}$ and $v_{i}$ represent linear transformation direction or projection direction, and the values of $\lambda_{i}$ are equal to projection of variance to the original vectors projection direction $u_{i}$ or $v_{i}$. The number of singular values depends on the balance between performance and complexity.

\section{E. Parameter Extraction Algorithm}

Predistortion system as shown in figure 2, in actual engineering, the error $e(n)$ of predistortion signals $z(n)$ and its estimated signals $\hat{z}(n)$ can't completely ideal is equal to zero, only according to certain rules of the best minimize error signal. Here adopts the minimum mean square error criterion, minimize the squared error of the LS algorithm to train predistortion device parameters [10].

Predistortion of the input-output relationship, see Equation (2), for convenience of description, transcribe into matrix form:

$$
z=X a
$$

Where:

$$
\begin{gathered}
z=[z(0), \ldots, z(N-1)]^{T} \\
a=\left[a_{1,0}, a_{3,0}, \ldots, a_{K, 0}, \ldots, a_{1, Q}, \ldots, a_{K, Q}\right]^{T}
\end{gathered}
$$

Define:

$$
x_{k q}(n)=x(n-q)|x(n-q)|^{k-1}
$$

Then

$$
X=\left[x_{1,0}, x_{3,0}, \ldots, x_{K, 0}, \ldots, x_{1, Q}, \ldots, x_{K, Q}\right]
$$

Equation (2) applied to the predistorter training module $A$, the input-output relationship is given by:

$$
\hat{z}(n)=\sum_{k=1}^{K} \sum_{q=0}^{Q} a_{k q} \frac{y(n-q)}{G}\left|\frac{y(n-q)}{G}\right|^{k-1}
$$

Equation (9) can be written in the form of matrix:

$$
\hat{z}=Y a
$$

The estimated error between the output of the module $\hat{z}(n)$ and the output of the predistorter $z(n)$ is:

$$
e(n)=z(n)-\hat{z}(n)
$$


Using the least squares theory, the optimal solution for the memory polynomial coefficients of the predistorter training module is:

$$
\hat{a}=\left(Y^{H} Y\right)^{-1} Y^{H} z
$$

The LS algorithm steps are based on SVD decomposition as follows:

- According to the observation sampling data obtained from the amplifier output terminal, construct matrix $\mathrm{Y}$, and with the ideal predistortion signal $\mathrm{z}(\mathrm{n})$ configure desired vector $\mathrm{z}$.

- Calculate singular value decomposition of matrix $Y$, yielding the rank of matrix $Y^{H} Y$ nonzero singular values $\sigma_{1}, \sigma_{2}, \cdots, \sigma_{K}$, and corresponding right singular vectors $q_{1}, q_{2}, \cdots, q_{K}$.

- Calculate the coefficients $\hat{a}$ using Equation (12). $\left(\hat{a}=\sum_{i=0}^{K} \frac{q_{i}}{\sigma_{i}^{2}}\left(q_{i}^{H} \theta\right), \theta=Y^{H} z\right)$

\section{SimUlation AND COMPARISON}

OFDM modulation signal with bandwidth of $8 \mathrm{MHz}$ is the input. The model of PA is the memory polynomials, whose order is 7 and memory depth is 2 .

\section{A. PSD Performance with DPD}

- Singular value decomposition takes 12 singular values. Nearly $35 \mathrm{~dB}$ improvement is obtained as shown in Figure 3.

- Singular value decomposition takes 8 singular values. Nearly $28 \mathrm{~dB}$ improvement is obtained as shown in Figure 4.

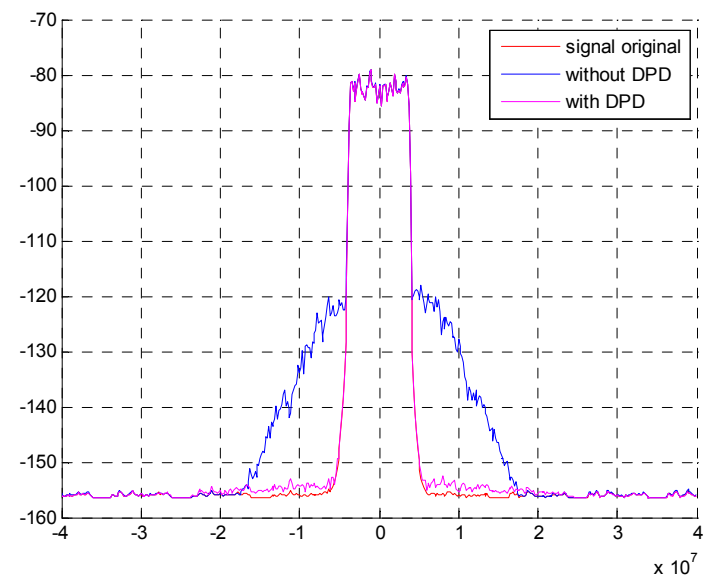

Figure 3. PSD with 12 singular values of DPD

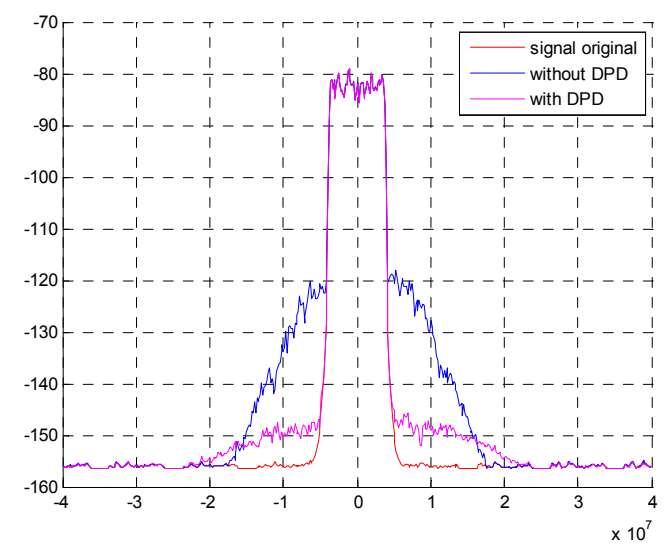

Figure 4. PSD with 8 singular values of DPD

\section{B. AM-AM and AM-PM Performance}

Nonlinear characteristics of PA can be represented by their AM-AM and AM-PM performance.

- Singular value decomposition takes 12 singular values. AM-AM and AM-PM get improved with DPD which are showed in Figure 5 and Figure 6

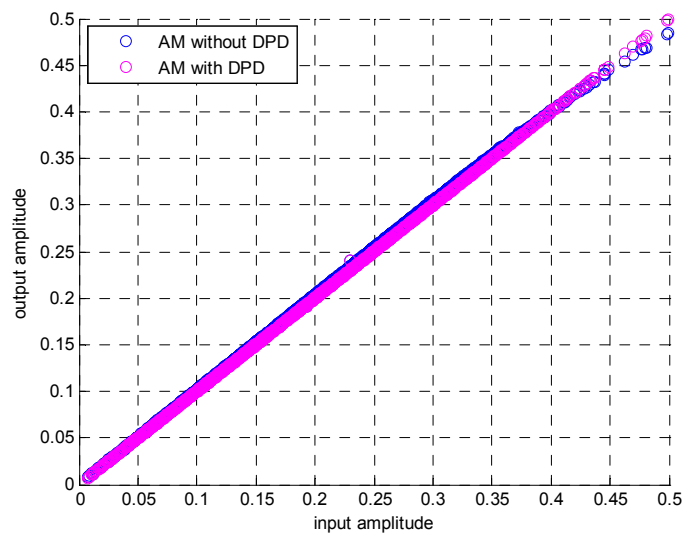

Figure 5. AM-AM with 12 singular values

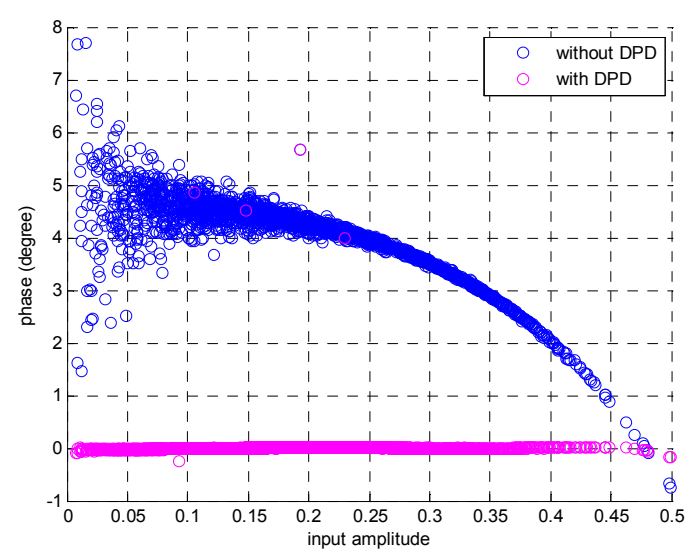

Figure 6. AM-PM with 12 singular values 
- Singular value decomposition takes 8 singular values. AM-AM and AM-PM get improved with DPD which are showed in Figure 7 and Figure 8

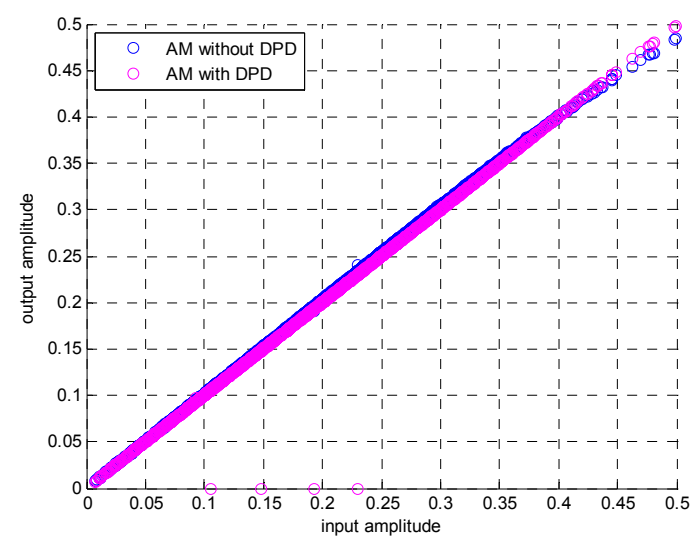

Figure 7. AM-PM with 8 singular values

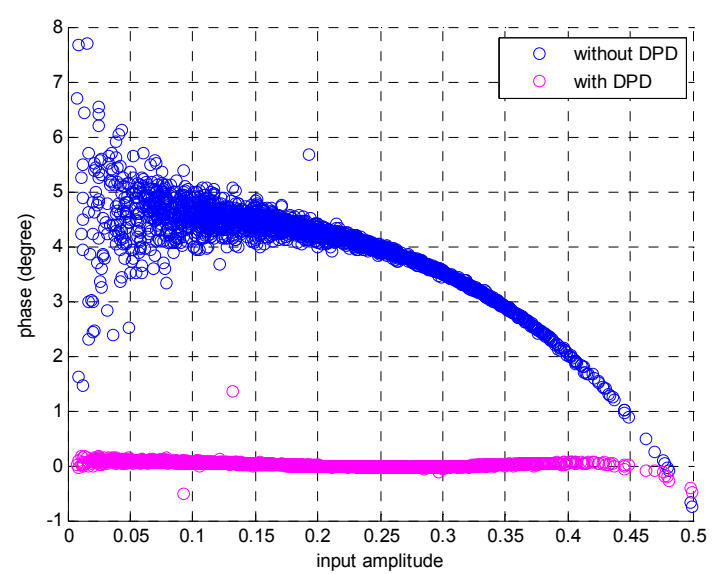

Figure 8. AM-PM with 8 singular values

\section{CONCLUSION}

Simulation results show that compared with traditional LS algorithm, LS with singular value decomposition applied in this paper can reduce the adaptive algorithm complexity significantly and contribute approximate predistortion performance.

\section{REFERENCES}

[1] J. F. Sevic and J. Staudinger, Simulation of power amplifier adjacent channel power ratio for digital wireless communication systems, IEEE Veh. Technol. Conf., vol. 2, 1997, pp. 681-685.

[2] J Cha, J Yi, J Kim. Optimum Design of a Predistortion RF Power Amplifier for Multicarrier WCDMA Applications[J]. IEEE Trans. Microwave Theory Tech, 2004, vol. 52(2):655-546.

[3] Kenington P B. High Linearity RF Amplifier Design. Boston, MA: Artech House, 2000.

[4] Mandic D P, Obradovic D, Kuh K. A robust general normalized gradient descent algorithm//IEEE/SP 13th Workshop on Statistical Signal Processing, Paris, 2005:133-136

[5] Ai B, Zhong Z D, Zhu G, et al. A Novel Scheme for Power Amplifier Predistortion Based on Indirect Leaning Architecture. Wireless Personal Communications, 2008, 46(4):523-530

[6] Isaksson M. Behavioural modeling of radio frequency power amplifiers// An Evaluation of Some Block Structure and Neural Network Models, Uppsala,2005.

[7] J.H.K. Vuolevi, T Rahkonen, J.P.A. Manninen. Measurement technique for characterizing memory effects in RF power amplifiers [J]. IEEE Trans. On Microwave Theory Tech., 2001, 49(8):13831388.

[8] P. L. Gilabert, D. D. Silveira, G. Montoro, M. E. Gadringer, and E. Bertran, Heuristisc algorithms for power amplifier behavioral modelling, IEEE Microw. Wireless Compon. Lett., vol. 17, no. 10, pp.715-717, Oct. 2007.

[9] Bergqvist, G. Larsson , E. G. The Higher-Order Singular Value Decompsition: Theory and an Application[J], IEEE Signal Processing, 2010, vol. 27(3):151-154.

[10] Guan Lei, Optimmized Low-Complexity Implementation of Least Squares Based Model Extraction for Digital Predistortion of RF Power Amplifiers[J], IEEE trans. On Microwave Theory and Tech., 2012, vol. 60(3):594-603. 